\title{
Milnor invariants and the HOMFLYPT Polynomial
}

\author{
JEAN-BAPTISTE MEILHAN \\ AKIRA YASUHARA
}

\begin{abstract}
We give formulas expressing Milnor invariants of an $n$-component link $L$ in the 3 -sphere in terms of the HOMFLYPT polynomial as follows. If the Milnor invariant $\bar{\mu}_{L}(J)$ vanishes for any sequence $J$ with length at most $k$, then any Milnor $\bar{\mu}$-invariant $\bar{\mu}_{L}(I)$ with length between 3 and $2 k+1$ can be represented as a combination of HOMFLYPT polynomial of knots obtained from the link by certain band sum operations. In particular, the "first nonvanishing" Milnor invariants can be always represented as such a linear combination.
\end{abstract}

$57 \mathrm{M} 25,57 \mathrm{M} 27$

\section{Introduction}

J Milnor defined in $[19 ; 20]$ a family of link invariants, known as Milnor $\bar{\mu}$-invariants. Here, and throughout the paper, by a link we mean an oriented, ordered link in $S^{3}$. Roughly speaking, Milnor invariants encode the behaviour of parallel copies of each link component in the lower central series of the link group. Given an $n$-component link $L$ in $S^{3}$, Milnor invariants are specified by a sequence $I$ of (possibly repeating) indices from $\{1, \ldots, n\}$. The length of the sequence is called the length of the Milnor invariant. It is known that Milnor invariants of length two are just linking numbers. However in general, Milnor invariant $\bar{\mu}_{L}(I)$ is only well-defined modulo the greatest common divisor $\Delta_{L}(I)$ of all Milnor invariants $\bar{\mu}_{L}(J)$ such that $J$ is obtained from $I$ by removing at least one index and permuting the remaining indices cyclicly. This indeterminacy comes from the choice of the meridian curves generating the link group. Equivalently, it comes from the indeterminacy of representing the link as the closure of a string link; see Habegger and Lin [7]. See Section 4 for the definitions.

Recall that the HOMFLYPT polynomial of a knot $K$ is of the form $P(K ; t, z)=$ $\sum_{k=0}^{N} P_{2 k}(K ; t) z^{2 k}$, and denote by $P_{0}^{(l)}(K)$ the $l$-th derivative of $P_{0}(K ; t) \in \mathbb{Z}\left[t^{ \pm 1}\right]$ evaluated at $t=1$. Denote by $\left(\log P_{0}(K)\right)^{(n)}$ the $n$-th derivative of $\log P_{0}(K ; t)$ evaluated at $t=1$. Since $P_{0}(K ; 1)=1$ and $P_{0}^{(1)}(K)=0$, we have

$$
\left(\log P_{0}\right)^{(n)}=P_{0}^{(n)}+\sum_{k_{1}+\cdots+k_{m}=n} n_{\left(k_{1}, \ldots, k_{m}\right)} P_{0}^{\left(k_{1}\right)} \ldots P_{0}^{\left(k_{m}\right)},
$$


where the sum runs over all $k_{1}, \ldots, k_{m}$ such that $k_{1}+\cdots+k_{m}=n\left(k_{i} \geq 2\right)$, and where $n_{\left(k_{1}, \ldots, k_{m}\right)} \in \mathbb{Z}$. For example, one can check that $\left(\log P_{0}\right)^{(n)}=P_{0}^{(n)}$ for $n=1,2,3$, and that $\left(\log P_{0}\right)^{(4)}=P_{0}^{(4)}-3\left(P_{0}^{(2)}\right)^{2}$.

In this paper, we show the following. If Milnor invariant $\bar{\mu}_{L}(J)$ vanishes for any sequence $J$ with length at most $k$, then any Milnor $\bar{\mu}$-invariant $\bar{\mu}_{L}(I)$ with length $m+1(3 \leq m+1 \leq 2 k+1)$ is given by a linear combination of $\left(\log P_{0}\right)^{(m)}$ invariants of knots obtained from the link by certain band sum operations.

For simplicity, we first state the formula for Milnor link-homotopy invariants $\bar{\mu}(I)$, ie such that the sequence $I$ has no repeated index. Let $L=\bigcup_{i=1}^{n} L_{i}$ be an $n-$ component link in $S^{3}$. Let $I=i_{1} i_{2} \ldots i_{m}$ be a sequence of $m$ distinct elements of $\{1, \ldots, n\}$. Let $B_{I}$ be an oriented $(2 m)$-gon, and denote by $p_{j}(j=1, \ldots, m)$ a set of $m$ nonadjacent edges of $B_{I}$ according to the boundary orientation. Suppose that $B_{I}$ is embedded in $S^{3}$ such that $B_{I} \cap L=\bigcup_{j=1}^{m} p_{j}$, and such that each $p_{j}$ is contained in $L_{i_{j}}$ with opposite orientation. We call such a disk an $I$-fusion disk for $L$. For any subsequence $J$ of $I$, we define the oriented knot $L_{J}$ as the closure of $\left(\left(\bigcup_{i \in\{J\}} L_{i}\right) \cup \partial B_{I}\right) \backslash\left(\left(\bigcup_{i \in\{J\}} L_{i}\right) \cap B_{I}\right)$, where $\{J\}$ is the subset of $\{1, \ldots, n\}$ of all indices appearing in the sequence $J$.

Theorem 1.1 Let $L$ be an $n$-component link in $S^{3}(n \geq 3)$ with vanishing Milnor link-homotopy invariants of length up to $k$. Then for any sequence $I$ of $(m+1)$ distinct elements of $\{1, \ldots, n\}(3 \leq m+1 \leq 2 k+1)$ and for any $I$-fusion disk for $L$, we have

$$
\left.\bar{\mu}_{L}(I) \equiv \frac{-1}{m ! 2^{m}} \sum_{J<I}(-1)^{|J|}\left(\log P_{0}\left(L_{J}\right)\right)^{(m)}\left(\bmod \Delta_{L}(I)\right)\right),
$$

where the sum runs over all subsequences $J$ of $I$, and where $|J|$ denotes the length of the sequence $J$.

This generalizes widely a result of M Polyak for Milnor's triple linking number $\bar{\mu}(123)$ [22]. There are several other known results relating Milnor invariants of (string) links to the Alexander polynomial; for example see Cochran [3], Levine [13], Masbaum and Vaintrob [16], Meilhan [17], Murasugi [21] and Traldi [24; 25] (note in particular that the results of $[13 ; 16 ; 17 ; 22]$ make use of closure-type operations). The relationship to quantum invariants is also known via the Kontsevich integral; see Habegger and Masbaum [8].

We emphasize that our assumption that the link has vanishing Milnor link-homotopy invariants of length up to $k$ is essential in order to compute its Milnor invariants of length up to $2 k+1$ using our formula. See the example at the end of this paper, which 
shows that Milnor link-homotopy invariants of length 4 are not given by the formula in Theorem 1.1 if there are nonvanishing linking numbers.

As noted above, only the first nonvanishing Milnor invariants of a link are well-defined integer-valued invariants. The following is in some sense a refinement of Theorem 1.1 for the first nonvanishing Milnor invariants.

Theorem 1.2 Let $L$ be an $n$-component link in $S^{3}(n \geq 3)$ with vanishing Milnor link-homotopy invariants of length up to $k(\geq 2)$. Then for any sequence $I$ of $(k+1)$ distinct elements of $\{1, \ldots, n\}$ and for any $I$-fusion disk for $L$, we have

$$
\bar{\mu}_{L}(I)=\frac{-1}{k ! 2^{k}} \sum_{J<I}(-1)^{|J|} P_{0}^{(k)}\left(L_{J}\right) \in \mathbb{Z} .
$$

Theorem 1.2 implies that all Milnor link-homotopy invariants of a link $L$ vanish if and only if all linking numbers of $L$ vanish and $\sum_{J<I}(-1)^{|J|} P_{0}^{(k)}\left(L_{J}\right)=0$ for all $k$ $(2 \leq k \leq n-1)$ and for all nonrepeated sequences $I$ of length $k+1$.

We remark that since the HOMFLYPT polynomial of knots is preserved by mutation, by Theorem 1.2, the first nonvanishing Milnor link-homotopy invariants are also preserved (cf Cha [2, Theorems 1.4 and 1.5]).

Theorem 1.1 and Theorem 1.2 generalize as follows. Let $L=\bigcup_{i=1}^{n} L_{i}$ be an $n-$ component link in $S^{3}$, and let $I=i_{1} i_{2} \ldots i_{m}$ be a sequence of $m$ elements of $\{1, \ldots, n\}$, where each element $i$ appears exactly $r_{i}$ times. Denote by $D_{I}(L)$ the $m$-component link obtained from $L$ as follows.

- Replace each string $L_{i}$ by $r_{i}$ zero-framed parallel copies of it, labeled from $L_{(i, 1)}$ to $L_{\left(i, r_{i}\right)}$. If $r_{i}=0$ for some index $i$, simply delete $L_{i}$.

- Let $D_{I}(L)=L_{1}^{\prime} \cup \cdots \cup L_{m}^{\prime}$ be the $m$-string link $\cup_{i, j} L_{(i, j)}$ with the order induced by the lexicographic order of the index $(i, j)$. This ordering defines a bijection $\varphi:\left\{(i, j) \mid 1 \leq i \leq n, 1 \leq j \leq r_{i}\right\} \rightarrow\{1, \ldots, m\}$.

We also define a sequence $D(I)$ of elements of $\{1, \ldots, m\}$ without repeated index as follows. First, consider a sequence of elements of $\left\{(i, j) \mid 1 \leq i \leq n, 1 \leq j \leq r_{i}\right\}$ by replacing each $i$ in $I$ with $(i, 1), \ldots,\left(i, r_{i}\right)$ in this order. For example if $I=12231$, we obtain the sequence $(1,1),(2,1),(2,2),(3,1),(1,2)$. Next replace each term $(i, j)$ of this sequence with $\varphi((i, j))$. Hence we have $D(12231)=13452$.

Theorem 1.3 Let $L$ be an $n$-component link in $S^{3}$ with vanishing Milnor invariants of length up to $k$. Let $I$ be a sequence of $(m+1)$ possibly repeating elements of $\{1, \ldots, n\}(3 \leq m+1 \leq 2 k+1)$. 
(i) For any $D(I)$-fusion disk for $D_{I}(L)$, we have

$$
\bar{\mu}_{L}(I) \equiv \frac{-1}{m ! 2^{m}} \sum_{J<D(I)}(-1)^{|J|}\left(\log P_{0}\left(D_{I}(L) J\right)\right)^{(m)}\left(\bmod \Delta_{L}(I)\right) .
$$

(ii) If $m=k(\geq 2)$, then for any $D(I)$-fusion disk for $D_{I}(L)$, we have

$$
\bar{\mu}_{L}(I)=\frac{-1}{k ! 2^{k}} \sum_{J<D(I)}(-1)^{|J|} P_{0}^{(k)}\left(D_{I}(L)_{J}\right) \in \mathbb{Z} .
$$

Theorem 1.3 follows directly from Theorems 1.1 and 1.2, since Milnor proved in [20, Theorem 7] that $\bar{\mu}_{D_{I}(L)}(D(I))=\bar{\mu}_{L}(I)$ (note that $\Delta_{L}(I)=\Delta_{D_{I}(L)}(D(I))$, again as a consequence of [20, Theorem 7]).

Theorem 1.3 implies the following corollary.

Corollary 1.4 All Milnor invariants of a link $L$ vanish if and only if all linking numbers of $L$ are zero and $\sum_{J<D(I)}(-1)^{|J|} P_{0}^{(k)}\left(D_{I}(L)_{J}\right)=0$ for all $k(\geq 2)$ and for all sequences $I$ with length $k+1$.

The rest of the paper is organized as follows. In Section 2, we review some elements of the theory of claspers, which is the main tool in proving our main results. In Section 3, we recall some properties of the HOMFLYPT polynomial of knots. In Section 4, we review Milnor invariants and string links and give a few lemmas. Section 5 is devoted to the proof of Theorem 1.1. In Section 6, we prove Theorem 1.2 and show, as a consequence, how to use the HOMFLYPT polynomial to distinguish string links up to link-homotopy. The paper is concluded by a simple example which illustrates the necessity of the assumptions required in our results.

Convention 1.5 In this paper, given a sequence $I$ of elements of $\{1, \ldots, n\}$, the notation $J<I$ will be used for any subsequence $J$ of $I$, possibly empty or equal to $I$ itself. By $J \lesseqgtr I$, we mean any subsequence $J$ of $I$ that is not $I$ itself. We will use the notation $\{I\}$ for the subset of $\{1, \ldots, n\}$ formed by all indices appearing in the sequence $I$, and $|I|$ will denote the length of the sequence $I$.

Acknowledgements The authors thank Kazuo Habiro for useful discussions. The first author is supported by the French ANR research project ANR-11-JS01-00201 and by the JSPS Invitation Fellowship for Research in Japan (Short Term) number S-10127. The second author is partially supported by a JSPS Grant-in-Aid for Scientific Research (C) number 23540074 .

This work is dedicated to Professor Shin'ichi Suzuki on his 70th birthday. 


\section{Some elements of clasper theory}

The primary tool in the proofs of our results is the theory of claspers. We recall here the main definitions and properties of this theory that will be useful in subsequent sections. For a general definition of claspers, we refer the reader to Habiro [9].

Definition 2.1 Let $L$ be a (string) link. A surface $G$ embedded in $S^{3}$ (or $D^{2} \times[0,1]$ ) is called a graph clasper for $L$ if it satisfies the following three conditions:

(1) $G$ is decomposed into disks and bands, called edges, each of which connects two distinct disks.

(2) The disks have either 1 or 3 incident edges, and are called leaves or vertices respectively.

(3) $G$ intersects $L$ transversely, and the intersections are contained in the union of the interiors of the leaves.

In particular, if a graph clasper $G$ is a disk, we call it a tree clasper.

Throughout this paper, the drawing convention for claspers are those of [9, Figure 7], unless otherwise specified.

The degree of a connected graph clasper $G$ is defined as half of the number of vertices and leaves. A tree clasper of degree $k$ is called a $C_{k}-$ tree. Note that a $C_{k}$-tree has exactly $(k+1)$ leaves.

A graph clasper for a (string) link $L$ is simple if each of its leaves intersects $L$ at exactly one point.

Let $G$ be a simple graph clasper for an $n$-component (string) link $L$. The index of $G$ is the collection of all integers $i$ such that $G$ intersects the $i$-th component of $L$. For example, if $G$ intersects component 3 twice and components 2 and 5 once, and is disjoint from all other components of $L$, then its index is $\{2,3,5\}$.

Given a graph clasper $G$ for a (string) link, there is a procedure to construct a framed link, in a regular neighbourhood of $G$. There is thus a notion of surgery along $G$, which is defined as surgery along the corresponding framed link. In particular, surgery along a simple $C_{k}$-tree is a local move as illustrated in Figure 1.

The $C_{k}$-equivalence is the equivalence relation on (string) links generated by surgeries along connected graph claspers of degree $k$ and isotopies. Alternatively, the $C_{k}$-equivalence can be defined in term of "insertion" of elements of the $k$-th term of the lower central series of the pure braid group; see Stanford [23]. We use the notation $L \sim C_{k} L^{\prime}$ for $C_{k}$-equivalent (string) links $L$ and $L^{\prime}$. 


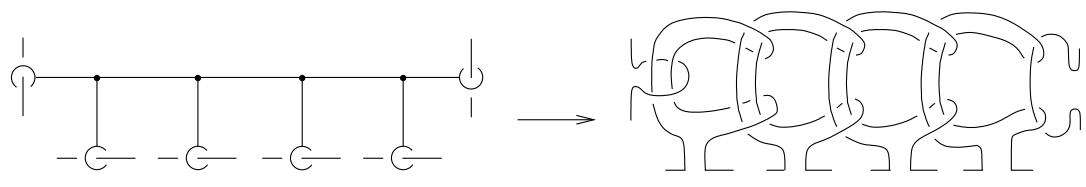

Figure 1. Surgery along a simple $C_{5}$-tree

It is known that the $C_{k}$-equivalence becomes finer as $k$ increases, and that two links are $C_{k}$-equivalent if and only if they are related by surgery along simple $C_{k}$-trees [9]. Moreover, it was shown by Goussarov [6] and Habiro [9] that this equivalence relation characterizes the topological information carried by finite type knot invariants. More precisely, it is shown in [6;9] that two knots are $C_{k}$-equivalent if and only if they cannot be distinguished by any finite type invariant of degree $<k$.

\subsection{Linear trees and planarity}

For $k \geq 3$, a $C_{k}$-tree $G$ having the shape of the tree clasper in Figure 1 is called a linear $C_{k}$-tree. The left-most and right-most leaves of $G$ in Figure 1 are called the ends of $G$.

Now suppose that the $G$ is a linear $C_{k}$-tree for some knot $K$, and denote its ends by $f$ and $f^{\prime}$. Then the remaining $(k-1)$ leaves of $G$ can be labelled from 1 to $(k-1)$, by travelling along the boundary of the $\operatorname{disk}^{1} G$ from $f$ to $f^{\prime}$ so that all leaves are visited. We say that $G$ is planar if, when travelling along $K$ from $f$ to $f^{\prime}$, either following or against the orientation, the labels of the leaves met successively are strictly increasing. See Figure 2 for an example.
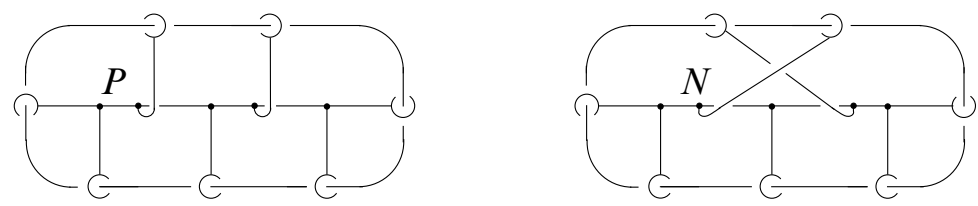

Figure 2. The $C_{6}$-tree $P$ is planar for the unknot, while $N$ is nonplanar.

\subsection{Calculus of claspers for parallel claspers}

We shall need refinements of [9, Propositions 4.4 and 4.6] for parallel tree claspers.

\footnotetext{
${ }^{1}$ Recall that a clasper is an embedded surface: in particular, since $T$ is a tree clasper, the underlying surface is homeomorphic to a disk.
} 
Here, by parallel tree claspers we mean a family of $m$ parallel copies of a tree clasper $T$, for some $m \geq 1$. We call $m$ the multiplicity of the parallel clasper. Note that there is no ambiguity in the notion of parallel copies here, since for a tree clasper the underlying surface is homeomorphic to a disk.

Lemma 2.2 Let $m, k, k^{\prime} \geq 1$ be integers. Let $T$ be a parallel $C_{k}$-tree with multiplicity $m$ for a (string) link $L$, and let $T^{\prime}$ be a $C_{k^{\prime}}$-tree for $L$, disjoint from $T$.

(1) Let $\tilde{T} \cup \tilde{T}^{\prime}$ be obtained from $T \cup T^{\prime}$ by sliding a leaf $f^{\prime}$ of $T^{\prime}$ over $m$ parallel leaves of $T$ (see Figure 3(a)). Then $L_{T \cup T^{\prime}}$ is ambient isotopic to $L_{\tilde{T} \cup \tilde{T}^{\prime} \cup Y \cup C}$, where $Y$ denotes the parallel $C_{k+k^{\prime}}$-tree with multiplicity $m$ obtained by inserting a vertex $v$ in the edge $e$ of $T$ and connecting $v$ to the edge incident to $f^{\prime}$ as shown in Figure 3(a), and where $C$ is a disjoint union of $C_{k+k^{\prime}+1}$-trees for $L$.

(2) Let $\tilde{T} \cup \tilde{T}^{\prime}$ be obtained from $T \cup T^{\prime}$ by passing an edge of $T^{\prime}$ across $m$ parallel edges of $T$ (see Figure 3(b)). Then $L_{T \cup T^{\prime}}$ is ambient isotopic to $L_{\widetilde{T} \cup \widetilde{T^{\prime}} \cup H \cup C}$, where $H$ denotes the parallel $C_{k+k^{\prime}+1}$-tree with multiplicity $m$ obtained by inserting vertices in both edges, and connecting them by an edge as shown in Figure 3(b), and where $C$ is a disjoint union of $C_{k+k^{\prime}+2}$-trees for $L$.
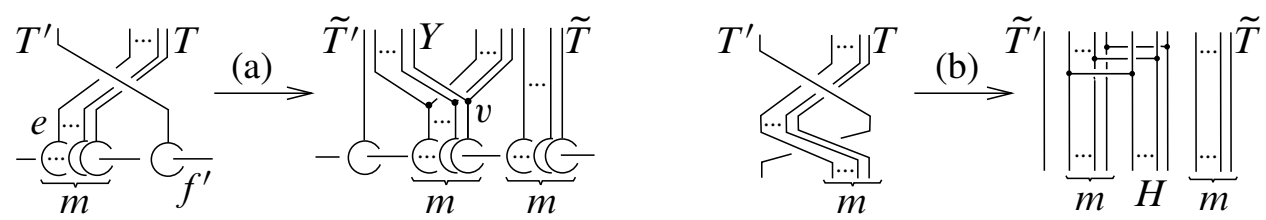

Figure 3. Leaf slide and crossing change involving parallel tree claspers

This result is well-known for $m=1$. The general case is easily proved using the arguments of the proof of [9, Propositions 4.4 and 4.6], respectively.

Remark 2.3 Notice that, following the proofs of [9, Propositions 4.4 and 4.6], the index of each of the tree claspers involved in Lemma 2.2 can be determined from those of $T$ and $T^{\prime}$ as follows. We have that the index of $\widetilde{T}$ is equal to the index of $T$, the index of $\widetilde{T}^{\prime}$ is equal to the index of $T^{\prime}$, and the indices of $Y, H$ and each connected component of $C$ are equal to the union of the indices of $T$ and $T^{\prime}$.

\section{The HOMFLYPT polynomial}

In this section, we recall the definition of the HOMFLYPT polynomial, and mention a few useful examples and properties. 
The HOMFLYPT polynomial $P(L ; t, z) \in \mathbb{Z}\left[t^{ \pm 1}, z^{ \pm 1}\right]$ of an oriented link $L$ is defined by the formulas

(1) $P(U ; t, z)=1$,

(2) $t^{-1} P\left(L_{+} ; t, z\right)-t P\left(L_{-} ; t, z\right)=z P\left(L_{0} ; t, z\right)$,

where $U$ denotes the unknot and where $L_{+}, L_{-}$and $L_{0}$ are three links that are identical except in a 3-ball where they look as follows:

$$
L_{+}=\nwarrow \lambda, \quad L_{-}=\nwarrow \lambda, \quad L_{0}=\nwarrow \nearrow \text { ( }
$$

In particular, the HOMFLYPT polynomial of an $r$-component link $K$ is of the form

$$
P(K ; t, z)=\sum_{k=1}^{N} P_{2 k-1-r}(K ; t) z^{2 k-1-r},
$$

where $P_{2 k-1-r}(K ; t) \in \mathbb{Z}\left[t^{ \pm 1}\right]$ is called the $(2 k-1-r)$-th coefficient polynomial of $K$. Furthermore, the lowest degree coefficient polynomial of $K$ is given by

$$
P_{1-r}(K ; t)=t^{2 \operatorname{Lk}(L)}\left(t^{-1}-t\right)^{r-1} \prod_{i=1}^{r} P_{0}\left(K_{i} ; t\right),
$$

where $K_{i}$ denotes the $i$-th component of $K$, and where $\operatorname{Lk}(L):=\sum_{i<j} \operatorname{lk}\left(L_{i}, L_{j}\right)$; see [14, Proposition 22].

Denote by $P_{k}^{(l)}(L)$ the $l$-th derivative of $P_{k}(L ; t)$ evaluated at $t=1$. It was proved by Kanenobu and Miyazawa that $P_{k}^{(l)}$ is a finite type invariant of degree $k+l$ [12]. In particular, $P_{0}^{(l)}$ is of degree $l$, and thus is an invariant of $C_{l+1}$-equivalence.

It is well-known that the HOMFLYPT polynomial of knots is multiplicative under connected sum. Thus the same holds for the lowest degree coefficient polynomial $P_{0}$, and in general, for any integer $n$ and any two oriented knots $K$ and $K^{\prime}$, we have

$$
P_{0}^{(n)}\left(K \sharp K^{\prime}\right)=P_{0}^{(n)}(K)+P_{0}^{(n)}\left(K^{\prime}\right)+\sum_{k=1}^{n-1}\left(\begin{array}{l}
n \\
k
\end{array}\right) P_{0}^{(k)}(K) P_{0}^{(n-k)}\left(K^{\prime}\right) .
$$

If, moreover, we assume that the knot $K$ is $C_{n}$-equivalent to the unknot, then we have

$$
P_{0}^{(n)}\left(K \sharp K^{\prime}\right)=P_{0}^{(n)}(K)+P_{0}^{(n)}\left(K^{\prime}\right),
$$

since $P_{0}^{(k)}$ is an invariant of $C_{k+1}$-equivalence, for all $k$.

In general, a simple way to derive an additive knot invariant from the coefficient polynomial $P_{0}$ is to take its $\log$. (Since $P_{0}(K ; t)$ is in $\mathbb{Z}\left[t^{ \pm 1}\right]$ and $P_{0}(K ; 1)=1$ for 
any knot $K, \log P_{0}(K ; t)$ can be regarded as a smooth function defined on an open interval which contains 1.) Indeed, we have that, for any two oriented knots $K$ and $K^{\prime}$,

$$
\left(\log P_{0}\right)\left(K \sharp K^{\prime} ; t\right)=\left(\log P_{0}\right)(K ; t)+\left(\log P_{0}\right)\left(K^{\prime} ; t\right) .
$$

Denote by $\left(\log P_{0}(K)\right)^{(n)}$ the $n$-th derivative of $\log P_{0}(K ; t)$ evaluated at $t=1$. As mentioned in the introduction, $\left(\log P_{0}(K)\right)^{(n)}$ is equal to $P_{0}(K)^{(n)}$ plus a sum of products of $P_{0}(K)^{(k)}$ 's with $k<n$. So we see that $\left(\log P_{0}\right)^{(n)}$ is an additive finite type knot invariant of degree $n$, and thus is an invariant of $C_{n+1}$-equivalence.

The following simple example shall be useful later.

Lemma 3.1 Let $n \geq 1$, and let $\varepsilon=\left(\varepsilon_{0}, \varepsilon_{1}, \ldots, \varepsilon_{n}, \varepsilon_{n+1}\right) \in\{-1,1\}^{n+2}$. Let $K_{n}^{\varepsilon}$ be the knot represented in Figure 4. Then

$$
\left(\log P_{0}\left(K_{n}^{\varepsilon}\right)\right)^{(n+1)}=P_{0}^{(n+1)}\left(K_{n}^{\varepsilon}\right)=(-1)^{n} 2^{n+1}(n+1) ! \prod_{i=0}^{n+1} \varepsilon_{i} .
$$

Notice that $K_{n}^{\varepsilon}$ is $C_{n+1}$-equivalent to the unknot, and that for all $k \leq n$, we thus have $\left(\log P_{0}\left(K_{n}^{\varepsilon}\right)\right)^{(k)}=P_{0}^{(k)}\left(K_{n}^{\varepsilon}\right)=0$.

Proof Let us prove the second equality. We first prove the formula for the knot $K_{n}^{+}:=K_{n}^{(1, \ldots, 1)}$, by induction on $n$. Since $K_{1}^{+}$is the trefoil, we have $P_{0}^{(2)}\left(K_{1}^{+}\right)=-8$. Suppose that $n>1$. Clearly, changing the crossing $c$ of $K_{n}^{+}$yields the unknot (see Figure 4). Hence

$$
P_{0}\left(K_{n}^{+} ; t\right)=t^{2}+t P_{-1}\left(L_{n} ; t\right),
$$

where $L_{n}=K_{1} \cup K_{2}$ is the 2-component link represented on the right-hand side of Figure 4. Notice that $K_{1}$ is an unknot, while $K_{2}$ is a copy of the knot $K_{n-1}^{+}$. Hence
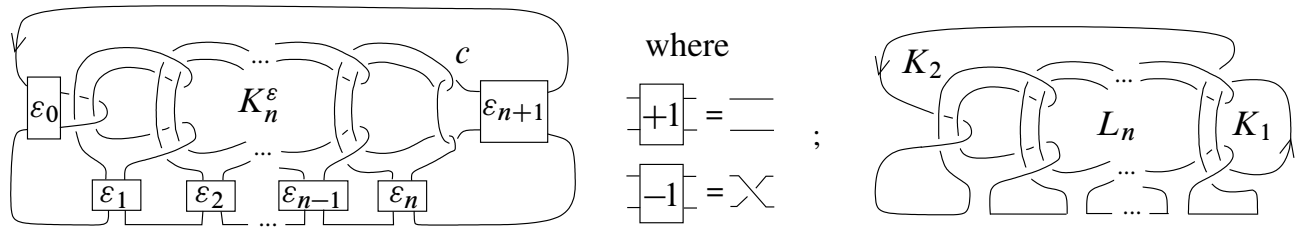

Figure 4. The knot $K_{n}^{\varepsilon}$ and the 2-component link $L_{n}$

by Equation (3-1) we have

$$
P_{0}\left(K_{n}^{+} ; t\right)=t^{2}+\left(1-t^{2}\right) P_{0}\left(K_{n-1}^{+} ; t\right) .
$$


By differentiating this equation $(n+1)$ times and evaluating at $t=1$, we obtain

$$
P_{0}^{(n+1)}\left(K_{n}^{+}\right)=-2(n+1) P_{0}^{(n)}\left(K_{n-1}^{+}\right)-n(n+1) P_{0}^{(n-1)}\left(K_{n-1}^{+}\right) .
$$

Since $K_{n-1}^{+}$is $C_{n}$-equivalent to the unknot, we see that $P_{0}^{(n-1)}\left(K_{n-1}^{+}\right)=0$. Hence we have

$$
P_{0}^{(n+1)}\left(K_{n}^{+}\right)=-2(n+1) P_{0}^{(n)}\left(K_{n-1}^{+}\right) .
$$

The induction hypothesis implies $P_{0}^{(n+1)}\left(K_{n}^{+}\right)=(-1)^{n} 2^{n+1}(n+1)$ !.

Now, notice that in general $K_{n}^{\varepsilon}$ is obtained from the unknot by surgery along the linear $C_{n+1}$-tree represented in Figure 5. It follows from [9, Claim on page 36] that

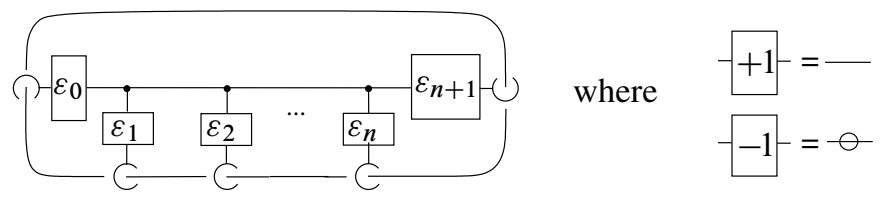

Figure 5. Here, a $\ominus$ on an edge represents a negative half-twist.

$K_{n}^{\varepsilon} \sharp K_{n}^{+}$is $C_{n+2}$-equivalent to the unknot (resp. to $K_{n}^{+} \sharp K_{n}^{+}$) if $\prod_{i=0}^{n+1} \varepsilon_{i}$ is equal to -1 (resp. to $=1$ ). Since $P_{0}^{(n+1)}$ is an invariant of $C_{n+2}$-equivalence and the knot $K_{n}^{\varepsilon}$ is $C_{n+1}$-equivalent to the unknot for any $\varepsilon \in\{-1,1\}^{n+2}$, we have

$$
\left(1+\prod_{i=0}^{n+1} \varepsilon_{i}\right) P_{0}^{(n+1)}\left(K_{n}^{+}\right)=P_{0}^{(n+1)}\left(K_{n}^{\varepsilon} \sharp K_{n}^{+}\right)=P_{0}^{(n+1)}\left(K_{n}^{\varepsilon}\right)+P_{0}^{(n+1)}\left(K_{n}^{+}\right)
$$

Hence we have

$$
P_{0}^{(n+1)}\left(K_{n}^{\varepsilon}\right)=P_{0}^{(n+1)}\left(K_{n}^{+}\right) \prod_{i=0}^{n+1} \varepsilon_{i} .
$$

The second equality follows.

Recall that $\left(\log P_{0}\right)^{(n+1)}$ is given by the sum of $P_{0}^{(n+1)}$ and a combination of $P_{0}^{(k)}$ 's with $k \leq n$. Since the knot $K_{n}^{\varepsilon}$ is $C_{n+1}$-equivalent to the unknot, the first equality follows.

We note from the above proof that Lemma 3.1 gives the variation of $\left(\log P_{0}\right)^{(n+1)}$ and $P_{0}^{(n+1)}$ under surgery along a planar linear $C_{n+1}$-tree for the unknot. On the other hand, the HOMFLYPT polynomial does not change under surgery along a nonplanar tree clasper, as follows from a formula of Kanenobu [11].

Lemma 3.2 Let $T$ be a nonplanar linear tree clasper for a knot $K$. Then $P_{0}\left(K_{T} ; t\right)=$ $P_{0}(K ; t)$. 
Proof We may assume that $K_{T}$ and $K$ are given by identical diagrams, except in a disk where they differ as illustrated in Figure 6. Let $L\left[\varepsilon_{2}, \ldots, \varepsilon_{n}\right]\left(\varepsilon_{j} \in\{-1,1\}, j=2, \ldots, n\right)$

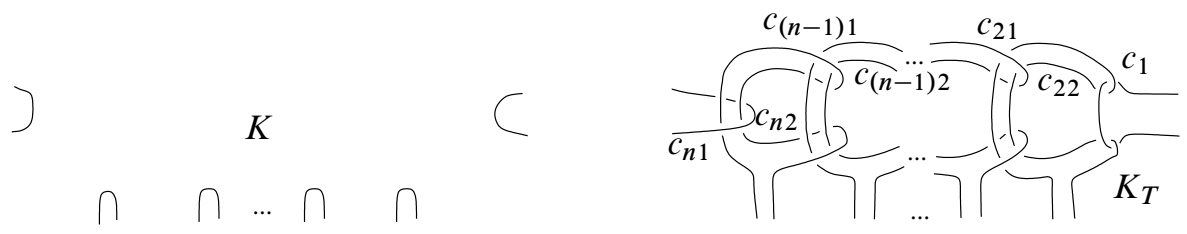

Figure 6. The knots $K$ and $K_{T}$

be the link obtained from $K_{T}$ by smoothing the crossing $c_{1}$, and

(i) smoothing the crossing $c_{j 1}$ if $\varepsilon_{j}=1$, or

(ii) changing the crossing $c_{j 1}$ and smoothing the crossing $c_{j 2}$ if $\varepsilon_{j}=-1$.

Kanenobu showed that if all links $L\left[\varepsilon_{2}, \ldots, \varepsilon_{n}\right]$ have less than $n+1$ components, then $P_{0}\left(K_{T} ; t\right)=P_{0}(K ; t)$; see [11, (3.9)]. Moreover, Kanenobu showed how to estimate the number of components of $L\left[\varepsilon_{2}, \ldots, \varepsilon_{n}\right]$ using a kind of a chord diagram which corresponds to the smoothed crossings. More precisely, the chord diagram associated to $L\left[\varepsilon_{2}, \ldots, \varepsilon_{n}\right]$ represents the $n$-singular knot obtained from $K_{T}$ by changing the crossing $c_{1}$ and each crossing $c_{j 1}$ (resp. $c_{j 2}$ ) such that $\varepsilon_{j}=1$ (resp. $\varepsilon_{j}=-1$ ) into double points. Kanenobu showed that $L\left[\varepsilon_{2}, \ldots, \varepsilon_{n}\right]$ has $n+1$ components if and only if the associated chord diagram contains no intersection among the chords; see the proof of [11, Lemma (3.7)]. If $T$ is nonplanar, then it is not hard to see that, for any $\left(\varepsilon_{j} \in\{-1,1\}, j=1,2, \ldots, n\right)$, the corresponding chord diagram contains such an intersection. Thus we have the conclusion.

Remark 3.3 Lemma 3.1 and Lemma 3.2 are related to the main results of Kanenobu [11] and Horiuchi [10].

\section{Milnor invariants}

\subsection{A short definition}

Given an $n$-component link $L$ in $S^{3}$, denote by $\pi$ the fundamental group of $S^{3} \backslash L$, and by $\pi_{q}$ the $q$-th subgroup of the lower central series of $\pi$. We have a presentation of $\pi / \pi_{q}$ with $n$ generators, given by a choice of meridian $m_{i}$ of the $i$-th component of $L, i=1, \ldots, n$. So the longitude $\lambda_{j}$ of the $j$-th component of $L(1 \leq j \leq n)$ is expressed modulo $\pi_{q}$ as a word in the $m_{i}$ 's (abusing notation, we still denote 
this word by $\left.\lambda_{j}\right)$. The Magnus expansion $E\left(\lambda_{j}\right)$ of $\lambda_{j}$ is the formal power series in noncommuting variables $X_{1}, \ldots, X_{n}$ obtained by substituting $1+X_{i}$ for $m_{i}$ and $1-X_{i}+X_{i}^{2}-X_{i}^{3}+\cdots$ for $m_{i}^{-1}, 1 \leq i \leq n$.

Let $I=i_{1} i_{2} \ldots i_{k-1} j$ be a sequence of elements of $\{1, \ldots, n\}$. Denote by $\mu_{L}(I)$ the coefficient of $X_{i_{1}} \cdots X_{i_{k-1}}$ in the Magnus expansion $E\left(\lambda_{j}\right)$. The Milnor invariant $\bar{\mu}_{L}(I)$ is the residue class of $\mu_{L}(I)$ modulo the greatest common divisor of all $\mu_{L}(J)$ such that $J$ is obtained from $I$ by removing at least one index and permuting the remaining indices cyclicly $[19 ; 20]$. The indeterminacy comes from the choice of the meridians $m_{i}$. Equivalently, it comes from the indeterminacy of representing the link as the closure of a string link [7]. Let us recall below the definition of these objects.

\subsection{String links}

Let $n \geq 1$, and let $D^{2} \subset R^{2}$ be the unit disk equipped with $n$ marked points $x_{1}, \ldots, x_{n}$ in its interior, lying on the diameter on the $x$-axis of $R^{2}$. An $n$-string link, or $n-$ component string link, is the image of a proper embedding $\bigsqcup_{i=1}^{n}[0,1]_{i} \rightarrow D^{2} \times[0,1]$ of the disjoint union $\bigsqcup_{i=1}^{n}[0,1]_{i}$ of $n$ copies of $[0,1]$ in $D^{2} \times[0,1]$, such that for each $i$, the image of $[0,1]_{i}$ runs from $\left(x_{i}, 0\right)$ to $\left(x_{i}, 1\right)$. Each string of an $n$-string link is equipped with an (upward) orientation. The $n$-string link $\left\{x_{1}, \ldots, x_{n}\right\} \times[0,1]$ in $D^{2} \times[0,1]$ is called the trivial $n-$ string link and is denoted by $\mathbf{1}_{n}$.

For each marked point $x_{i} \in D^{2}$, there is a point $y_{i}$ on $\partial D^{2}$ in the upper half of $R^{2}$ such that the segment $p_{i}=x_{i} y_{i}$ is vertical to the $x$-axis, as illustrated in Figure 8. Given an $n$-string link $L=\bigcup_{i=1}^{n} L_{i}$ in $D^{2} \times[0,1] \subset R^{2} \times[0,1]$, the closure $\widehat{L}$ of $L$ is the $n$-component link defined by $\widehat{L}=\bigcup_{i=1}^{n} \hat{L}_{i}=L \cup\left(\bigcup_{i=1}^{n}\left(p_{i} \times\{0,1\}\right) \cup\left(y_{i} \times[0,1]\right)\right)$.

The set of isotopy classes of $n$-string links fixing the endpoints has a monoid structure, with composition given by the stacking product and with the trivial $n$-string link $\mathbf{1}_{n}$ as unit element. Given two $n$-string links $L$ and $L^{\prime}$, we denote their product by $L \cdot L^{\prime}$, which is obtained by stacking $L^{\prime}$ above $L$ and reparametrizing the ambient cylinder $D^{2} \times[0,1]$.

Habegger and Lin showed that Milnor invariants are actually well defined integer-valued invariants of string links [7]. (We refer the reader to [7] or Yasuhara [26] for a precise definition of Milnor invariants $\mu(I)$ of string links.) Furthermore, Milnor invariants of length $k$ are known to be finite-type invariants of degree $k-1$ for string links by Bar-Natan [1] and Lin [15]. As a consequence, Milnor invariants of length $k$ for string links are invariants of $C_{k}$-equivalence. Habiro showed that the same actually holds for Milnor invariants of links [9]. 


\subsection{Some results}

It was shown by Habegger and Lin that Milnor invariants without repeated indices classify string links up to link-homotopy [7]. Here, the link-homotopy is the equivalence relation generated by self-crossing changes. In [27], the second author gave an explicit representative for the link-homotopy class of any $n$-string link in terms of linear tree claspers. We shall make use of this representative in this paper, and recall its definition below.

Let $\mathcal{J}_{k}$ denote the set of all sequences $j_{0} j_{1} \ldots j_{k}$ of $k+1$ nonrepeating integers from $\{1, \ldots, n\}$ such that $j_{0}<j_{m}<j_{k}$ for all $m$. Let $i_{0} i_{1} \ldots i_{k}$ be a sequence of $(k+1)$ integers from $\{1, \ldots, n\}$ such that $i_{0}<i_{1}<\cdots<i_{k-1}<i_{k}$, and let $a_{J}$ be a permutation of $\left\{i_{1}, \ldots, i_{k-1}\right\}$. Then $J=i_{0} a_{J}\left(i_{1}\right) \cdots a_{J}\left(i_{k-1}\right) i_{k}$ is in $\mathcal{J}_{k}$ (and all elements of $\mathcal{J}_{k}$ can be realized in this way). Let $T_{J}$ be the simple linear $C_{k}$-tree for $\mathbf{1}_{n}$ as illustrated in Figure 7. Here, $a_{J}$ is the unique positive $k$-braid which defines the permutation $a_{J}$ and such that every pair of strings crosses at most once. In the figure, we also implicitly assume that all edges of $T_{J}$ overpass all components of $\mathbf{1}_{n}$. (This assumption is crucial in the computation of Milnor invariants.) Let $\bar{T}_{J}$ be the

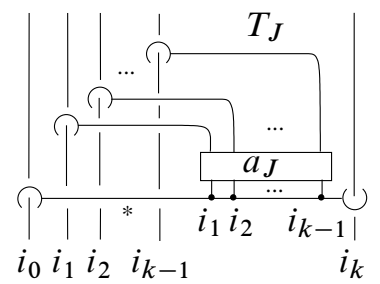

Figure 7. The $C_{k}$-trees $T_{J}$ and $\bar{T}_{J}$

$C_{k}$-tree obtained from $T_{J}$ by inserting a positive half-twist in the $*$-marked edge; see Figure 7. Denote respectively by $V_{J}$ and $V_{J}^{-1}$ the $n$-string links obtained from $\mathbf{1}_{n}$ by surgery along $T_{J}$ and $\bar{T}_{J}$. This notation is justified by the fact that, for any $J$ in $\mathcal{J}_{k}$, the string link $V_{J} \cdot V_{J}^{-1}$ is $C_{k+1}$-equivalent to the trivial one [9].

Theorem 4.1 [27] Any $n$-string link $L$ is link-homotopic to $l=l_{1} \cdots l_{n-1}$, where

$$
l_{i}=\prod_{J \in \mathcal{J}_{i}} V_{J}^{x_{J}}, \text { where } \quad x_{J}=\mu_{l_{i}}(J)= \begin{cases}\mu_{L}(J) & \text { if } i=1, \\ \mu_{L}(J)-\mu_{l_{1} \cdots l_{i-1}}(J) & \text { if } i \geq 2 .\end{cases}
$$

Remark 4.2 The above statement slightly differs from the one in [27]. There, another family of $C_{k}$-trees is used in place of $T_{J}$ and $\bar{T}_{J}$. However, the present statement is 
shown by the exact same arguments as for [27, Theorem 4.3], since for any $J, J^{\prime} \in \mathcal{J}_{k}$ $(k \geq 1)$, we have

$$
\mu_{V_{J}}\left(J^{\prime}\right)= \begin{cases}1 & \text { if } J=J^{\prime} \\ 0 & \text { otherwise }\end{cases}
$$

(compare with [27, Lemma 4.1]).

For the representative $l=l_{1} \cdots l_{n-1}$ above, we have the following lemmas.

Lemma 4.3 Let $I<12 \ldots n$ with $|I|=m \leq n$. Then,

$$
\mu_{l}(I) \equiv x_{I}\left(\bmod \operatorname{gcd}\left\{x_{J} \mid J \lesseqgtr I\right\}\right) .
$$

Moreover, for all $k<m-1$, we have

$$
\mu_{l_{1} \cdots l_{k}}(I) \equiv 0\left(\bmod \operatorname{gcd}\left\{x_{J}|J \lesseqgtr I,| J \mid \leq k+1\right\}\right) .
$$

Proof By [18, Lemma 3.3] and Theorem 4.1, we have $\mu_{l}(I)=\mu_{l_{1} \cdots l_{m-2}}(I)+$ $\mu_{l_{m-1}}(I)=\mu_{l_{1} \cdots l_{m-2}}(I)+x_{I}$. Hence (4-3) implies (4-2), and it suffices to prove Equation (4-3).

Note that, for an $n$-string link $L=\bigcup_{i=1}^{n} L_{i}$, we have $\mu_{I}(L)=\mu_{I}\left(\bigcup_{i \in\{I\}} L_{i}\right)$. Hence we may assume that $I=12 \ldots m$ and that $l$ is an $m$-string link.

The result is shown by an analysis of the Magnus expansion of a longitude of each "building block" $V_{J}^{x_{J}}$, for all $k<m-1$ and all $J \in \mathcal{J}_{k}$. Since we are aiming at computing Milnor invariant $\mu(I)$, we compute up to terms $O(I)$ involving monomials $X_{i_{1}} X_{i_{2}} \cdots X_{i_{p}}$ such that $i_{1} i_{2} \ldots i_{p}$ is not a subsequence of $12 \ldots(m-1)$. Note that $O(I)$ includes any monomial where some variable appears at least twice, as well as any monomial involving $X_{m}$. For a subset $\{K\}$ of $\{I\}$, we will also use the notation $M(\{K\})$ for a sum of terms involving monomials such that all $X_{j}(j \in\{K\})$ appear exactly once in each monomial.

Let $J=j_{0} j_{1} \ldots j_{k} \in \mathcal{J}_{k}$, for some $k<m-1$. Let $j$ be an index in $I$, and denote by $\lambda_{j}$ the $j$-th longitude of $V_{J}^{ \pm 1}$. Notice that all monomials appearing in the Magnus expansion $E\left(\lambda_{j}\right)$ are in the variables $X_{i}$ such that $i \in\{J\}$, since all edges of both $T_{J}$ and $\bar{T}_{J}$ overpass all components of $\mathbf{1}_{n}$. There are three cases:

(i) If $j<j_{0}$ or $j_{k}<j$, then clearly we have $E\left(\lambda_{j}\right)=1$.

(ii) If $j \in\{J\}$, since all Milnor invariants of $V_{J}^{ \pm 1}$ with length at most $k$ vanish,

$$
E\left(\lambda_{j}\right)=1+M(\{J\} \backslash\{j\})+M(\{J\})+O(I) .
$$


(iii) If $j \notin\{J\}$ and $j_{0}<j<j_{k}$, then since $V_{J}^{ \pm 1} \backslash$ (ith component) is trivial for any $i \in\{J\}$, all (nontrivial) monomials appearing in $E\left(\lambda_{j}\right)$ contain all variables $X_{i}(i \in\{J\})$. Hence we have a Magnus expansion of the form

$$
E\left(\lambda_{j}\right)=1+M(\{J\})+O(I) .
$$

Summarizing all three cases, we have

$$
\text { (4-4) } E\left(\lambda_{j}\right)= \begin{cases}1 & \text { if } j<j_{0} \text { or } j_{k}<j, \\ 1+M(\{J\} \backslash\{j\})+M(\{J\})+O(I) & \text { if } j \in\{J\}, \\ 1+M(\{J\})+O(I) & \text { if } j \notin\{J\} \text { and } j_{0}<j<j_{k} .\end{cases}
$$

Now let us consider the stacking product $V_{J}^{ \pm 1} \cdot V_{J_{\sim}}^{ \pm 1}$. The Magnus expansion of the $j$-th longitude of $V_{J}^{ \pm 1} \cdot V_{J}^{ \pm 1}$ is given by $E\left(\lambda_{j}\right) E\left(\tilde{\lambda}_{j}\right)$, where $\lambda_{j}$ is the $j$-th longitude of $V_{J}^{ \pm 1}$ and $E\left(\tilde{\lambda_{j}}\right)$ is obtained from $E\left(\lambda_{j}\right)$ by replacing $X_{i}$ with $E\left(\lambda_{i}\right)^{-1} X_{i} E\left(\lambda_{i}\right)$ for each $i \in\{I\}$. By (4-4), we have

$$
E\left(\lambda_{i}\right)^{-1} X_{i} E\left(\lambda_{i}\right)= \begin{cases}X_{i} & \text { if } i<j_{0} \text { or } j_{k}<i, \\ X_{i}+M(\{J\})+O(I) & \text { if } i \in\{J\}, \\ X_{i}+M(\{J\} \cup\{i\})+O(I) & \text { if } i \notin\{J\} \text { and } j_{0}<i<j_{k} .\end{cases}
$$

This implies that the Magnus expansion of the $j$-th longitude of $V_{J}^{ \pm 1} \cdot V_{J}^{ \pm 1}$ is given by

$$
E\left(\lambda_{j}\right) E\left(\tilde{\lambda_{j}}\right)= \begin{cases}1 & \text { if } j<j_{0} \text { or } j_{k}<j, \\ E\left(\lambda_{j}\right) E\left(\lambda_{j}\right)+M(\{J\})+O(I) & \text { if } j \in\{J\}, \\ 1+M(\{J\})+O(I) & \text { if } j \notin\{J\} \text { and } j_{0}<j<j_{k} .\end{cases}
$$

Generalizing this argument, we obtain that the Magnus expansion of the $j$-th longitude $\lambda_{J, j}$ in $V_{J}^{x_{J}}$ is given by

$$
E\left(\lambda_{J, j}\right)= \begin{cases}1 & \text { if } j<j_{0} \text { or } j_{k}<j, \\ E\left(\lambda_{j}\right)^{\left|x_{J}\right|}+M(\{J\})+O(I) & \text { if } j \in\{J\}, \\ 1+M(\{J\})+O(I) & \text { if } j \notin\{J\} \text { and } j_{0}<j<j_{k} .\end{cases}
$$

Let us now focus on the case $j=m$. There are two cases to consider.

- If $m$ is not in $\{J\}$, then necessarily $j_{k}<m$ and by (4-5) we have that the Magnus expansion of the $m$-th longitude $\lambda_{J, m}$ in $V_{J}^{x_{J}}$ is 1 .

- If $m$ is in $\{J\}$, then necessarily $j_{k}=m$. Since $j_{0}$ is the smallest integer in $\{J\}$, any monomial in $M(\{J\} \backslash\{m\})$ whose leftmost variable is not $X_{j_{0}}$ belongs to $O(I)$. Moreover, each term in $M(\{J\})$ involves the variable $X_{m}$, and hence belongs to $O(I)$. So (4-1) and (4-4) show that the Magnus expansion of the $m$-th longitude of $V_{J}^{ \pm 1}$ is $E\left(\lambda_{m}\right)=1 \pm X_{j_{0}} \cdots X_{j_{k-1}}+O(I)$. Equation (4-5) then implies that the Magnus expansion of the $m$-th longitude $\lambda_{J, m}$ in $V_{J}^{x_{J}}$ is given by $1+x_{J} X_{j_{0}} \cdots X_{j_{k-1}}+O(I)$. 
Summarizing, for $j=m$ we obtain that

$$
E\left(\lambda_{J, m}\right)= \begin{cases}1 & \text { if } m \notin\{J\}, \\ 1+O(I)\left(\bmod x_{J}\right) & \text { if } m \in\{J\} \text { and } J<I, \\ 1+O(I) & \text { otherwise. }\end{cases}
$$

We can now complete the computation of $\mu_{l_{1} \cdots l_{k}}(I)$. Since the Magnus expansion of the $m$-th longitude of $l_{1} \cdots l_{k}$ is obtained from a product of $E\left(\lambda_{J, m}\right)$ 's $(\{J\} \subset\{I\}, J \in$ $\bigcup_{s=1}^{k} \mathcal{J}_{s}$ ) by replacing each variable $X_{i}$ with $X_{i}+\left(\right.$ monomials involving $X_{i}$ ), it is of the form

$$
1+O(I)\left(\bmod \operatorname{gcd}\left\{x_{J}|J \lesseqgtr I,| J \mid \leq k+1\right\}\right) .
$$

This implies that $\mu_{l_{1} \cdots l_{k}}(I) \equiv 0\left(\bmod \operatorname{gcd}\left\{x_{J}|J \lesseqgtr I| J \mid, \leq k+1\right\}\right)$, as desired.

Lemma 4.4 Let $I<12 \ldots n$ with $|I|=m \leq n$. Then

$$
\Delta_{l}(I)=\operatorname{gcd}\left\{x_{J} \mid J \lesseqgtr I\right\} .
$$

Proof The proof is by induction on $m$. For $m=3$, the result is clear since $x_{i j}=$ $\mu_{l_{1}}(i j)=\mu_{l}(i j)$ for any $i, j$. Now, let $m \geq 4$. It will be convenient to use the notation $\delta_{k}(I)$ for the set of all sequences of length $(m-k)$ obtained from $I$ by removing $k$ indices and permuting cyclicly. By definition,

$$
\Delta_{l}(I)=\operatorname{gcd}\left(\left\{\mu_{l}(J) \mid J \in \delta_{k}(I), k>1\right\} \cup\left\{\mu_{l}(J) \mid J \in \delta_{1}(I)\right\}\right) .
$$

By the induction hypothesis, we have that

$$
\begin{aligned}
\operatorname{gcd}\left\{\mu_{l}(J) \mid J \in \delta_{k}(I), k>1\right\} & =\operatorname{gcd}\left\{\Delta_{l}(J) \mid J \in \delta_{1}(I)\right\} \\
& =\operatorname{gcd}\left\{\Delta_{l}(J) \mid J \in \delta_{1}(I), J<I\right\} \\
& =\operatorname{gcd}\left\{x_{J^{\prime}} \mid J^{\prime} \lesseqgtr J, J \in \delta_{1}(I), J<I\right\} \\
& =\operatorname{gcd}\left\{x_{J^{\prime}}\left|J^{\prime}<I,\right| J^{\prime} \mid<m-1\right\} .
\end{aligned}
$$

On the other hand, by Lemma 4.3, for all $J \in \delta_{1}(I)(J<I)$ and for any sequence $\tau(J)$ obtained from $J$ by permuting cyclicly, we have

$$
\mu_{l}(\tau(J)) \equiv \mu_{l}(J) \equiv x_{J}\left(\bmod \operatorname{gcd}\left\{x_{J^{\prime}} \mid J^{\prime} \lesseqgtr J\right\}\left(=\Delta_{l}(J)\right)\right) .
$$

It follows that $\Delta_{l}(I)=\operatorname{gcd}\left\{x_{J}|J<I| J \mid, \leq m-1\right\}$, as desired.

\section{Proof of Theorem 1.1}

Let $L=\bigcup_{i=1}^{n} L_{i}$ be an $n$-component link in $S^{3}$, and let $I$ be a sequence of $(m+1)$ distinct elements of $\{1, \ldots, n\}$. It is sufficient to consider here the case $m+1=n$, 
since, if $m+1<n$, we have that $\bar{\mu}_{L}(I)=\bar{\mu}_{\bigcup_{i \in\{I\}} L_{i}}(I)$. We may further assume that $I=12 \ldots n$ without loss of generality. Indeed, for any permutation $I^{\prime}$ of $12 \ldots n$, we have that $\bar{\mu}_{L}\left(I^{\prime}\right)=\bar{\mu}_{L^{\prime}}(12 \ldots n)$, where $L^{\prime}$ is obtained from $L$ by reordering the components appropriately.

We first show how to reformulate the problem in terms of string links.

\subsection{Closing string links into knots}

Let $B_{I}$ be an $I$-fusion disk for $L$, as defined in the introduction. Up to isotopy, we may assume that the $2 n$-gon $B_{I}$ lies in the unit disk $D^{2}$ as shown in Figure 8 , where the edges $p_{j}$ are defined by $p_{j}=x_{j} y_{j}, 1 \leq j \leq n$. We may furthermore assume that $L \cup B_{I}$ lies in the cylinder $D^{2} \times[0,1]$, such that $B_{I} \subset\left(D^{2} \times\{0\}\right)$, and such that

$$
L \cap \partial\left(D^{2} \times[0,1]\right)=\bigcup_{j=1}^{n}\left(\left(p_{j} \times\{0\}\right) \cup\left(\left\{y_{j}\right\} \times[0,1]\right) \cup\left(p_{j} \times\{1\}\right)\right) .
$$

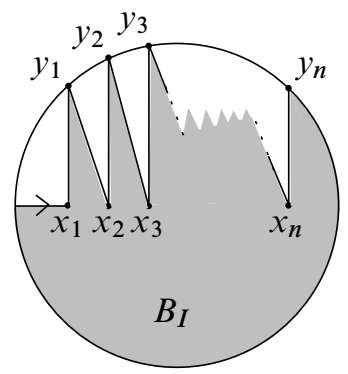

Figure 8 . The $2 n$-gon $B_{I}$ lying in the unit disk $D^{2}$

In this way, we obtain an $n$-string link $\sigma$ whose closure $\hat{\sigma}$ is the link $L$, by setting

$$
\sigma:=\overline{L \backslash\left(L \cap \partial\left(D^{2} \times[0,1]\right)\right)} \text {. }
$$

For example, Figure 9 represents a 3 -string link $\beta$ whose closure is the Borromean rings (there, the dotted part represents the intersection of the Borromean rings and $\left.\partial\left(D^{2} \times[0,1]\right)\right)$.

Given an $n$-string link $K=\bigcup_{i=1}^{n} K_{i}$ and any subsequence $J$ of $I=12 \ldots n$, we will denote by $K(J)$ the knot

$$
K(J):=\overline{\left(\left(\bigcup_{j \in\{J\}} \hat{K}_{j}\right) \cup \partial B_{I}\right) \backslash\left(\left(\bigcup_{j \in\{J\}} \hat{K}_{j}\right) \cap B_{I}\right)} .
$$




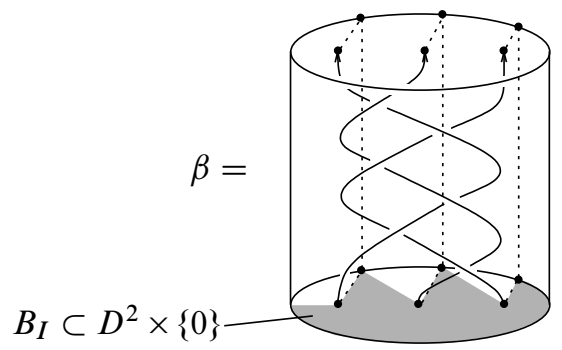

Figure 9. The string link $\beta$, whose closure is the Borromean rings

For example, Figure 10 represents the knots $\beta(13)$ and $\beta(123)$ obtained from the 3 -string link $\beta$ shown in Figure 9.

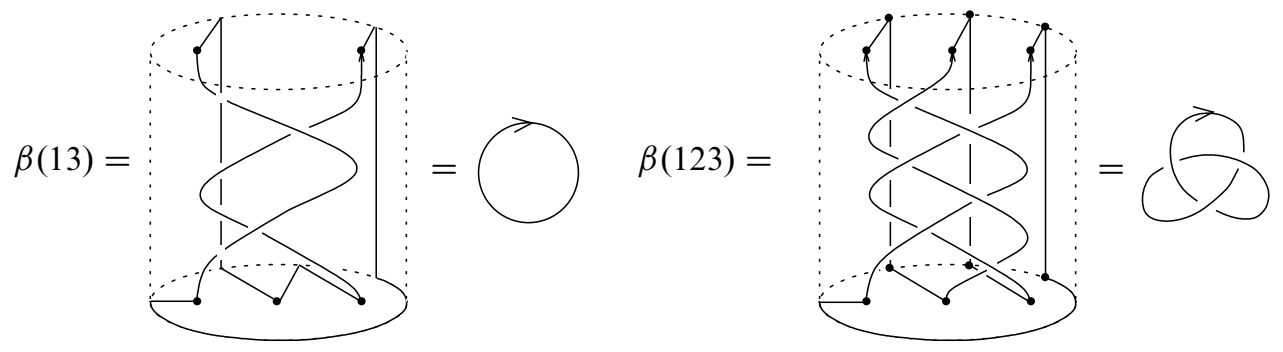

Figure 10. The knots $\beta(13)$ and $\beta(123)$ for the 3 -string link $\beta$

Note that $K(J)$ coincides with the knot $(\hat{K})_{J}$ defined in the introduction for the choice of $I$-fusion disk $B_{I}$ specified above.

Recall from Section 4.3 that, for any $k$ and any $J \in \mathcal{J}_{k}, V_{J}$ (resp. $V_{J}^{-1}$ ) denotes the $n$-string link obtained from $\mathbf{1}_{n}$ by surgery along the $C_{k}$-tree $T_{J}$ (resp. $\bar{T}_{J}$ ); see Figure 7. Denote by $t_{J}$ (resp. $\bar{t}_{J}$ ) the image of the $C_{k}$-tree $T_{J}$ (resp. $\bar{T}_{J}$ ) for $\mathbf{1}_{n}$ under taking the closure $V_{J}^{ \pm 1}(I)$. We observe that $t_{J}$ (resp. $\bar{t}_{J}$ ) is a planar tree clasper for the unknot if and only if $J<I$. In this case, note that $V_{J}(I)$ (resp. $\left.V_{J}^{-1}(I)\right)$ is the knot $K_{k-1}^{\varepsilon}$ of Figure 4 with $\varepsilon=(-,+, \ldots,+)$ (resp. for $\left.\varepsilon=(+,+, \ldots,+)\right)$. In particular, observe that $V_{I}^{x_{I}}(J)$ is the unknot for all $J \lesseqgtr I$ and that, by Lemma 2.2 (for $m=1$ ), the knot $V_{I}^{x_{I}}(I)$ is $C_{n}$-equivalent to the connected sum of $\left|x_{I}\right|$ copies of $V_{I}^{\varepsilon_{I}}(I)$, where $\varepsilon_{I}$ denotes the sign of $x_{I}$. By Lemmas 3.1 and 3.2 we thus have, for all $J \in \mathcal{J}_{n-1}$,

$$
\begin{aligned}
\left(\log P_{0}\left(V_{J}^{x_{J}}(I)\right)\right)^{(n-1)} & =P_{0}^{(n-1)}\left(V_{J}^{x_{J}}(I)\right) \\
& = \begin{cases}(-1)^{n-1} x_{I}(n-1) ! 2^{n-1} & \text { if } J=I, \\
0 & \text { otherwise }\end{cases}
\end{aligned}
$$




\subsection{Proof of Theorem 1.1}

Let $\sigma$ be the $n$-string link with closure $L$ defined in Section 5.1. By Theorem 4.1, $\sigma$ is link-homotopic to $l_{k} \cdots l_{n-1}$, where $l_{i}=\prod_{J \in \mathcal{J}_{i}} V_{J}^{x_{J}}$ is defined in Section 4.3, and with $n \leq 2 k+1$ (by our vanishing assumption on Milnor invariants). Hence $\sigma$ is obtained from $l_{k} \cdots l_{n-1}$ by surgery along a disjoint union $R_{1}$ of simple $C_{1}$-trees whose leaves intersect a single component of $l_{k} \cdots l_{n-1}$.

By Lemma 2.2, for all $J<I$, we have that

$$
\sigma(J) \sim_{C_{n}} l_{n-1}(J) \sharp\left(l_{k} \cdots l_{n-2}\right)_{R_{1}}(J) .
$$

Since $\left(\log P_{0}\right)^{(n-1)}$ is an invariant of $C_{n}$-equivalence for all $n$, it follows from the additivity property of $\left(\log P_{0}\right)$ that

$\left(\log P_{0}(\sigma(J))\right)^{(n-1)}=\left(\log P_{0}\left(l_{n-1}(J)\right)\right)^{(n-1)}+\left(\log P_{0}\left(\left(l_{k} \cdots l_{n-2}\right)_{R_{1}}(J)\right)\right)^{(n-1)}$.

The proof of the next lemma is postponed to Section 5.3.

\section{Lemma 5.1}

$$
\frac{-1}{(n-1) ! 2^{n-1}} \sum_{J<I}(-1)^{|J|}\left(\log P_{0}\left(\left(l_{k} \cdots l_{n-2}\right)_{R_{1}}(J)\right)\right)^{(n-1)} \equiv 0\left(\bmod \Delta_{L}(I)\right) .
$$

It follows from Equation (5-2) that

$$
\begin{aligned}
\frac{-1}{(n-1) ! 2^{n-1}} \sum_{J<I} & (-1)^{|J|}\left(\log P_{0}(\sigma(J))\right)^{(n-1)} \\
& \equiv \frac{-1}{(n-1) ! 2^{n-1}} \sum_{J<I}(-1)^{|J|}\left(\log P_{0}\left(l_{n-1}(J)\right)\right)^{(n-1)}\left(\bmod \Delta_{L}(I)\right) \\
& \equiv \frac{(-1)^{n-1}}{(n-1) ! 2^{n-1}}\left(\log P_{0}\left(V_{I}^{x_{I}}(I)\right)\right)^{(n-1)}\left(\bmod \Delta_{L}(I)\right) \\
& \equiv x_{I}\left(\bmod \Delta_{L}(I)\right)
\end{aligned}
$$

On the other hand, by Lemma 4.3 and Lemma 4.4, we have

$$
\mu_{L}(I)=\mu_{\sigma}(I)=\mu_{l_{k} \cdots l_{n-1}}(I) \equiv x_{I}\left(\bmod \Delta_{L}(I)\right),
$$

which completes the proof. 


\subsection{Proof of Lemma 5.1}

First, it is convenient to slightly modify the string link $\left(l_{k} \cdots l_{n-2}\right)_{R_{1}}$. For that purpose, we regard it as obtained from $\mathbf{1}_{n}$ by surgery along the disjoint union of tree claspers $G \cup R_{1}$, with

$$
G:=\bigcup_{i=k}^{n-2}\left(\bigcup_{J \in \mathcal{J}_{i}} T_{J}^{x_{J}}\right),
$$

where $T_{J}^{x_{J}}$ denotes $\left|x_{J}\right|$ parallel copies of $T_{J}$ (resp. $\bar{T}_{J}$ ) if $x_{J}>0$ (resp. if $\left.x_{J}<0\right)$. A tree clasper for $\mathbf{1}_{n}$ is said to be in good position if, in the usual diagram of $\mathbf{1}_{n}$, each component of $\mathbf{1}_{n}$ underpasses all edges of the tree clasper. For example, each component of $G$ is in good position (see Figure 7), whereas the components of $R_{1}$ may not be. However, by repeated applications of [9, Proposition 4.5] we have

$$
\left(\mathbf{1}_{n}\right)_{G \cup R_{1}} \sim_{C_{n}}\left(\mathbf{1}_{n}\right)_{G \cup \tilde{R}},
$$

where $\widetilde{R}$ is a disjoint union, disjoint from $G$, of simple tree claspers for $\mathbf{1}_{n}$ in good position and intersecting some component of $\mathbf{1}_{n}$ more than once.

We now close the string link $\left(\mathbf{1}_{n}\right)_{G \cup \widetilde{R}}$ using the sequence $I=12 \ldots n$, as explained in Section 5.1. It follows from Lemma 3.2 that, for all $J<I$, we have

$$
\left(\log P_{0}\left(\left(\mathbf{1}_{n}\right)_{G \cup \widetilde{R}}(J)\right)^{(n-1)}=\left(\log P_{0}\left(\left(\mathbf{1}_{n}\right)_{\tilde{G} \cup \widetilde{R}}(J)\right)^{(n-1)},\right.\right.
$$

where

$$
\widetilde{G}:=\bigcup_{i=k}^{n-2}\left(\bigcup_{J \in \mathcal{J}_{i} ; J<I} T_{J}^{x_{J}}\right) .
$$

In other words, we only need to consider those tree claspers $T_{J}$ and $\bar{T}_{J}$ with $J<I$, since only those become planar under closure. Moreover, since $\Delta_{L}(I)$ divides all $x_{J}$ with $J<I$, we can express each $T_{J}^{x_{J}}$ as a disjoint union of parallel tree claspers with multiplicity $\Delta_{L}(I){ }^{2}$ The $\operatorname{knot}\left(\mathbf{1}_{n}\right) \widetilde{G} \cup \widetilde{R}(I)$ is obtained from the unknot $U$ by surgery along a disjoint union of tree claspers

$$
F:=t \cup r, \quad t:=\bigcup_{i=1}^{q} t_{i}, \quad r:=\bigcup_{j=1}^{p} r_{j},
$$

for some integers $q, p$, where $r$ is the image of $\widetilde{R}$ under closure and where, for each $i=1, \ldots, p$, the clasper $t_{i}$ is a parallel family of $\Delta_{L}(I)$ copies of (the image under closure of) some $C_{m}$-tree $T_{J}$ or $\bar{T}_{J}$ with $J<I(k \leq m \leq n-1)$.

\footnotetext{
${ }^{2}$ Recall from Section 2.2 that a parallel tree clasper with multiplicity $k \geq 1$ is a family of $k$ parallel copies of a tree clasper.
} 
We need the following additional definition. A disjoint union $C_{1} \cup \cdots \cup C_{s}$ of $s \geq 1$ (possibly parallel) tree claspers of degree $<n$ for $U$ is called balanced if each tree $C_{j}$ is being assigned a subset $w\left(C_{j}\right)$ of $\{1, \ldots, n\}$, called weight, such that

$$
\left(\mathbf{1}_{n}\right)_{\tilde{G} \cup \widetilde{R}}(J) \sim C_{n} U_{\left(\cup_{w\left(C_{i}\right) \subset\{J\}} C_{i}\right)},
$$

for all $J<I$. For example, $F$ is balanced if we assign the index of each tree as weight. We say that a $C_{k}$-tree in a balanced family is repeated if its weight has at most $k$ elements, that is, if its weight is smaller than the number of leaves. For instance, all tree claspers $r_{j}(j=1, \ldots, p)$ are repeated.

Now, up to $C_{n}$-equivalence, we deform $U_{F}$ into a connected sum of knots obtained from $U$ by surgery along a single (possibly parallel) tree clasper. In other words, we will deform $F$ into a balanced union of localized tree claspers for $U$, ie tree claspers sitting in a 3-ball that intersects $U$ at a single strand and is disjoint from all other tree claspers. Since $U$ is the unknot, this deformation can be achieved, starting from $F$, by a sequence of only leaf slides and edge crossing changes; see Figure 3. By Lemma 2.2, performing such operations may introduce additional tree claspers up to $C_{n}$-equivalence. However, the following is easily verified.

Fact 5.2 Let $M \cup T \cup T^{\prime}$ be a balanced union of tree claspers for $U$, where $T \cup T^{\prime}$ is as in Lemma 2.2. If, in the statement of Lemma 2.2(1) (resp. of Lemma 2.2(2)), we assign the weights $w(T)$ and $w\left(T^{\prime}\right)$ to $\widetilde{T}$ and $\widetilde{T}^{\prime}$ respectively, and the weight $w(T) \cup w\left(T^{\prime}\right)$ to $Y$ (resp. $H$ ) and each connected component of $C$, then $M \cup \widetilde{T} \cup \widetilde{T}^{\prime} \cup Y \cup C$ (resp. $M \cup \widetilde{T} \cup \widetilde{T}^{\prime} \cup H \cup C$ ) is balanced. In particular, if the degrees of $T$ and $T^{\prime}$ are at least $(n-1) / 2$, where $n$ is the number of strands of $L$, then all tree claspers in $C$ are repeated.

We now start our localization process, which goes in three steps.

The first step consists in localizing all parallel tree claspers $t_{i}$. Consider, say, the parallel $C_{k_{1}}$-tree $t_{1}$. Then by assumption we have that $k_{1} \geq(n-1) / 2$, and Lemma 2.2 and Fact 5.2 imply that $U_{F} \sim C_{n} U_{t_{1}} \sharp U_{\left(F \backslash t_{1}\right) \cup F_{1}}$, where $F_{1}$ is a disjoint union of tree claspers of degree $>k_{1}$, which are either repeated trees or parallel tree claspers with multiplicity $\Delta(I)$. Using this argument repeatedly, we see that $F$ can be deformed into a balanced union of tree claspers $F^{\prime}=h_{1} \cup \cdots \cup h_{l} \cup r^{\prime}$, for some integer $l$, where $r^{\prime}$ is a disjoint union of repeated trees and each $h_{i}$ is a parallel tree clasper with multiplicity $\Delta_{L}(I)$, such that

$$
\left(\mathbf{1}_{n}\right)_{\tilde{G} \cup \widetilde{R}}(I)=U_{F} \sim_{C_{n}} U_{F^{\prime}}=U_{h_{1}} \sharp \cdots \sharp U_{h_{l}} \sharp U_{r^{\prime}} .
$$


In the second step, we "split" each parallel tree clasper into $\Delta_{L}(I)$ localized ones. Indeed, since each $h_{i}$ is a parallel family of $\Delta_{L}(I)$ copies of some tree clasper $h_{i}^{\prime}$, we can apply Lemma 2.2 (with $m=1$ ) and Fact 5.2 to deform $F^{\prime}$ into a balanced family

$$
F^{\prime \prime}=\bigcup_{i=1}^{l}(\underbrace{h_{i}^{\prime} \cup \cdots \cup h_{i}^{\prime}}_{\Delta_{L}(I) \text { times }}) \cup r^{\prime \prime},
$$

where for each $i$ the tree clasper $h_{i}^{\prime}$ has weight $w\left(h_{i}^{\prime}\right)=w\left(h_{i}\right)$ and where $r^{\prime \prime}$ is a disjoint union of repeated trees, such that

$$
U_{F^{\prime}} \sim_{C_{n}} U_{F^{\prime \prime}}=\left(\Delta_{L}(I) \times U_{h_{1}^{\prime}}\right) \sharp \cdots \sharp\left(\Delta_{L}(I) \times U_{h_{l}^{\prime}}\right) \sharp U_{r^{\prime \prime}} .
$$

(Here $\Delta_{L}(I) \times U_{h_{i}^{\prime}}$ denotes the connected sum of $\Delta_{L}(I)$ copies of $U_{h_{i}^{\prime}},(i=1, \ldots, l)$.

In the third and last step, we localize all repeated trees in $r^{\prime \prime}$. Note that, by Fact 5.2, performing a leaf slide or an edge crossing change between two repeated tree claspers only introduces new tree claspers that are also repeated. Hence $F^{\prime \prime}$ can be deformed into a balanced disjoint union of tree claspers

$$
X=\bigcup_{i=1}^{l}(\underbrace{h_{i}^{\prime} \cup \cdots \cup h_{i}^{\prime}}_{\Delta_{L}(I) \text { times }}) \cup \bigcup_{j=1}^{l^{\prime}} x_{j}
$$

for some integer $l^{\prime}$, where each $x_{j}$ is a repeated tree clasper, such that

$$
U_{F^{\prime \prime}} \sim_{C_{n}} U_{X}=\left(\Delta_{L}(I) \times U_{h_{1}^{\prime}}\right) \sharp \cdots \sharp\left(\Delta_{L}(I) \times U_{h_{l}^{\prime}}\right) \sharp U_{x_{1}} \sharp \cdots \sharp U_{x_{l^{\prime}}} .
$$

This concludes the localization process.

Now, since $X$ is balanced, and since $\log P_{0}$ is additive under connected sum (see Section 3), for any $J<I$ we have

$\left(\log P_{0}\left(\left(\mathbf{1}_{n}\right) \widetilde{G} \cup \widetilde{R}(J)\right)\right)^{(n-1)}$

$$
=\Delta_{L}(I) \sum_{w\left(h_{i}^{\prime}\right) \subset\{J\}}\left(\log P_{0}\left(U_{h_{i}^{\prime}}\right)\right)^{(n-1)}+\sum_{w\left(x_{j}\right) \subset\{J\}}\left(\log P_{0}\left(U_{x_{j}}\right)\right)^{(n-1)},
$$

where the first (resp. second) sum is over all tree claspers $h_{i}^{\prime}$ (resp. $x_{j}$ ) whose weight is contained in $\{J\}$. On the other hand, we have the following:

Claim 5.3 Let $g$ be a connected component of $X$.

(1) If $|w(g)|<n(=|I|)$, then

$$
\sum_{J<I, w(g) \subset\{J\}}(-1)^{|J|}\left(\log P_{0}\left(U_{g}\right)\right)^{(n-1)}=0 .
$$


(2) If $|w(g)|=n$ (ie $g=h_{i}^{\prime}$ for some $i$ and $g$ is a $C_{n-1}-$ tree), then

$$
\left(\log P_{0}\left(U_{g}\right)\right)^{(n-1)} \equiv 0\left(\bmod (n-1) ! 2^{n-1}\right) .
$$

Note that, since any connected component $g$ of $X$ has degree $<n$, we have that $|w(g)|<n$ if $g$ is repeated. Hence it follows from Claim 5.3 that

$$
\frac{-1}{(n-1) ! 2^{n-1}} \sum_{J<I}(-1)^{|J|}\left(\operatorname { l o g } P _ { 0 } \left(\left(\mathbf{1}_{n}\right) \widetilde{G} \cup \widetilde{R}^{(J)))^{(n-1)}} \equiv 0\left(\bmod \Delta_{L}(I)\right),\right.\right.
$$

which concludes the proof of Lemma 5.1.

Proof of Claim 5.3 (1) Since $w(g)<n$, there is an element $a \in\{I\}$ such that $a \notin w(g)$. We denote by $I \backslash a$ the sequence obtained from $I$ by deleting $a$. Then we have that

$$
\sum_{J<I, w(g) \subset\{J\}}(-1)^{|J|}=\sum_{J<I \backslash a, w(g) \subset\{J\}}(-1)^{|J|}+\sum_{J<I \backslash a, w(g) \subset\{J\} \cup\{a\}}(-1)^{|J|+1}=0,
$$

which implies the desired equality.

(2) Using the AS and IHX relations for tree claspers (see [6;9]), one can check that the knot $U_{g}$ is $C_{n}$-equivalent to a connected sum of knots $U_{g_{i}}$, where each $g_{i}$ is a linear $C_{n-1}$-tree which is either nonplanar or of the form shown in Figure 5. Since $\left(\log P_{0}\right)^{(n-1)}$ is an invariant of $C_{n}$-equivalence, the result then follows from Lemmas 3.1 and 3.2.

\section{First nonvanishing Milnor invariants and link-homotopy of string links}

We begin this section by proving Theorem 1.2. Most of the arguments follow very closely the proof of Theorem 1.1, and we therefore freely use the notions and results of the previous section.

\subsection{Proof of Theorem 1.2}

Let $L=\bigcup_{i=1}^{n} L_{i}$ be an $n$-component link in $S^{3}$ with vanishing Milnor link-homotopy invariants of length up to $k(3 \leq k+1 \leq n)$. Let $I$ be a sequence of $(k+1)$ distinct elements of $\{1, \ldots, n\}$. As in Section 5, we may assume without loss of generality that $k+1=n$ and that $I=12 \ldots n$. Following Section 5.1, we may also assume that the $2 n$-gon $B_{I}$ is chosen so that $L \cup B_{I}$ lies in the cylinder $D^{2} \times[0,1]$, such 
that $B_{I} \subset\left(D^{2} \times\{0\}\right)$ is as shown in Figure 8. Hence (5-1) defines an $n$-string link $\sigma$ whose closure is $L$.

By Theorem 4.1, the $n$-string link $\sigma$ is link-homotopic to $l_{n-1}=\prod_{J \in \mathcal{J}_{n-1}} V_{J}^{x_{J}}$ defined in Section 4.3. By applying the exact same arguments as in Section 5.3, there exists a disjoint union of tree claspers $R=r_{1} \cup \cdots \cup r_{p}$, with each being assigned a weight $w\left(r_{i}\right) \subset\{1, \ldots, n\}$, such that

- for each $i$, we have $\left|w\left(r_{i}\right)\right| \leq \operatorname{deg}\left(r_{i}\right)$,

- $\quad L_{J} \sim C_{n} l_{n-1}(J) \sharp U_{R_{J}}$ for all $J<I$, where $R_{J}=\bigcup_{w\left(r_{i}\right) \subset\{J\}} r_{i}$. (In particular, $R_{I}=R$ and $L_{I}$ is $C_{n}$-equivalent to $l_{n-1}(I) \sharp U_{R}$.)

Since $l_{n-1}$ is $C_{n-1}$-equivalent to $\mathbf{1}_{n}$, we have by Equation (3-2) that, for all $J<I$,

$$
P_{0}^{(n-1)}\left(L_{J}\right)=P_{0}^{(n-1)}\left(l_{n-1}(J)\right)+P_{0}^{(n-1)}\left(U_{R_{J}}\right) .
$$

The following lemma is proved below.

Lemma 6.1 $\sum_{J<I}(-1)^{|J|} P_{0}^{(n-1)}\left(U_{R_{J}}\right)=0$.

This lemma and Equation (5-2) imply that

$$
\begin{aligned}
\frac{-1}{(n-1) ! 2^{n-1}} \sum_{J<I}(-1)^{|J|} P_{0}^{(n-1)}\left(L_{J}\right) & =\frac{-1}{(n-1) ! 2^{n-1}} \sum_{J<I}(-1)^{|J|} P_{0}^{(n-1)}\left(l_{n-1}(J)\right) \\
& =\frac{-1}{(n-1) ! 2^{n-1}} \sum_{J<I}(-1)^{|J|} P_{0}^{(n-1)}\left(V_{I}^{x_{I}}(J)\right) \\
& =\frac{(-1)^{n-1}}{(n-1) ! 2^{n-1}} P_{0}^{(n-1)}\left(V_{I}^{x_{I}}(I)\right)=x_{I} .
\end{aligned}
$$

Lemma 4.3 completes the proof.

Proof of Lemma 6.1 We will show that the alternate sum

$$
\sum_{J<I}(-1)^{|J|} U_{R_{J}}
$$

is a linear combination of singular knots with $n$ double points. Since $P_{0}^{(n-1)}$ is a finite type invariant of degree $n-1$, this implies Lemma 6.1 .

We may assume without loss of generality that $\bigcup_{i} w\left(r_{i}\right)=\{1, \ldots, n\}$. Indeed, if there exists some $j \in\{1, \ldots, n\}$ such that $j \notin w\left(r_{i}\right)$ for all $i$, we can freely add a $C_{1}$-tree $c_{j}$ with weight $\{j\}$ such that $U_{R \cup c_{j}}=U_{R} \sharp U_{c_{j}}=U_{R} \sharp U$. 
For each $i=1, \ldots, p$, let $\operatorname{deg}\left(r_{i}\right)=d_{i}$. Consider the $\left(d_{i}+1\right)$-component trivial tangle which is the intersection of $U$ with a regular neighborhood of $r_{i}$. Then surgery along $r_{i}$ yields a $\left(d_{i}+1\right)$-component tangle $\beta^{i}=\beta_{0}^{i} \cup \cdots \cup \beta_{d_{i}}^{i}$. Note that $\beta^{i}$ is a Brunnian tangle [9]. Since $\beta^{i} \backslash \beta_{0}^{i}$ is trivial, there is a diagram of $\beta^{i}$ such that, for all $u=1, \ldots, d_{i}$, the component $\beta_{u}^{i}$ is a trivial arc that only crosses component $\beta_{0}^{i}$. Fix a diagram of $U_{R}$ that satisfies this condition for all $i=1, \ldots, p$. Now, let $w\left(r_{i}\right)=\left\{j_{1}, \ldots, j_{m_{i}}\right\} \subset\{1, \ldots, n\}$, with $m_{i} \leq d_{i}$, and for all $u \in\left\{1, \ldots, m_{i}\right\}$. Set

$$
\mathcal{S}_{i}\left(j_{u}\right):=\text { the set of all crossings where } \beta_{0}^{i} \text { underpasses } \beta_{u}^{i} \text {. }
$$

Note that this is only possible because $r_{i}$ satisfies $m_{i}=\left|w\left(r_{i}\right)\right| \leq d_{i}$. For all $j \in$ $\{1, \ldots, n\}$, set

$$
\mathcal{S}(j)=\bigcup_{i} \mathcal{S}_{i}(j)
$$

For any $J<I$, denote by $U_{R}[J]$ the knot obtained from $U_{R}$ by switching all crossings in $\bigcup_{j \in\{J\}} \mathcal{S}(j)$. Then $U_{R}[J]$ is obtained from $U$ by surgery along all $r_{i}$ such that $w\left(r_{i}\right) \cap\{J\}=\varnothing$, ie,

$$
U_{R}[J]=U_{R_{I \backslash J}}
$$

for any $J<I$, where $I \backslash J$ denotes the sequence obtained from $I$ by deleting all $j \in\{J\}$. (In particular, we have $U_{R}[\varnothing]=U_{R}$.) Hence we have

$$
\sum_{J<I}(-1)^{|J|} U_{R_{J}}=\sum_{J<I}(-1)^{|J|} U_{R}[I \backslash J]=\sum_{J<I}(-1)^{n-|J|} U_{R}[J],
$$

Clearly the alternate sum on the right-hand side, which involves knots that differ from one another by crossing changes on $n$ sets of crossings, can be written as a linear combination of singular knots with $n$ double points. This completes the proof of Lemma 6.1.

\subsection{Link-homotopy of string links}

In this section, we give several interesting consequences of Theorem 1.2 for Milnor invariants of string links.

We first define an analogue for string links of the band sum operations on links given in the introduction. Let $L$ be an $n$-string link. Recall from Section 4.2 and Section 5.1 that, for each $i=1, \ldots, n$, we pick a point $y_{i} \in \partial D^{2}$ and thus have a segment $p_{i}=x_{i} y_{i} \subset D^{2}$ (see Figure 8). Recall also that the closure of $L$ is defined by $\widehat{L}=\bigcup_{i=1}^{n} \hat{L_{i}}=L \cup\left(\bigcup_{i=1}^{n}\left(p_{i} \times\{0,1\}\right) \cup\left(y_{i} \times I\right)\right)$.

Let $I=i_{1} i_{2} \ldots i_{m+1}$ be a sequence of $m+1$ distinct integers in $\{1, \ldots, n\}$. We choose a $2(m+1)$-gon $B_{I}$ in $R^{2} \times(-\infty, 0]$ such that $B_{I} \cap\left(R^{2} \times\{0\}\right)=\bigcup_{i \in I}\left(p_{i} \times\{0\}\right)$ is 
a set of $m+1$ nonadjacent edges and $p_{i_{1}} \times\{0\}, \ldots, p_{i_{m+1}} \times\{0\}$ appear in this order along the oriented boundary of $B_{I}$. As in the introduction, for any subsequence $J$ of $I$, we can define an oriented knot $\widehat{L}_{J}$ as the closure of $\left(\left(\bigcup_{j \in\{J\}} \hat{L}_{j}\right) \cup \partial B_{I}\right) \backslash$ $\left(\left(\bigcup_{j \in\{J\}} \widehat{L}_{j}\right) \cap B_{I}\right)$.

Set

$$
f_{B_{I}}(L)=\frac{-1}{m ! 2^{m}} \sum_{J<I}(-1)^{|J|} P_{0}^{(m)}\left(\hat{L}_{J}\right) .
$$

This function depends on the choice of $B_{I}$. Hence, for every nonrepeated sequence $I$, we choose $B_{I}$ and fix it, to obtain an invariant of string links $f_{B_{I}}$.

The following is a string link version of Theorem 1.2.

Theorem 6.2 Let $L$ be an $n$-string link with vanishing Milnor link-homotopy invariants of length $\leq k(3 \leq k+1 \leq n)$. Then for any sequence $I$ of length $k+1$ without repeated indices, we have $\mu_{L}(I)=f_{B_{I}}(L)$.

Proof Let $\widehat{L}$ be the closure of $L$. Then $\bar{\mu}_{\widehat{L}}(J)=0$ for all sequence $J$ of length $\leq k$ without repeated indices, and $\bar{\mu}_{\hat{L}}(I)=\mu_{L}(I)$. The result then follows immediately from Theorem 1.2.

We now show how to use the $P_{0}$ polynomial to distinguish string links up to linkhomotopy.

Corollary 6.3 Two $n$-string links $L$ and $L^{\prime}$ are link-homotopic if and only if they have same linking numbers and $f_{B_{I}}\left(L \cdot \overline{L^{\prime}}\right)=0$ for all nonrepeated sequences $I$, where $\bar{L}^{\prime}$ denotes the horizontal mirror image of $L^{\prime}$ with the orientation reversed.

Proof The string link $\overline{L^{\prime}}$ is the inverse of $L^{\prime}$ under concordance, ie $L^{\prime} \cdot \overline{L^{\prime}}$ is concordant to the trivial string link. Since concordance of string links implies link-homotopy [4; 5], the two string links $L$ and $L^{\prime}$ are link-homotopic if and only if $L \cdot \overline{L^{\prime}}$ is link-homotopic to $\mathbf{1}_{n}$. (The result of [4;5] is given for links in $S^{3}$. However, it still holds for string links.) Corollary 6.3 follows from Theorem 6.2 and the fact that a string-link is link-homotopic to the trivial one if and only if all Milnor link-homotopy invariants of the link vanish [7].

For an $n$-string link $L$ and a sequence $I$ of possibly repeating elements of $\{1, \ldots, n\}$, we can define a nonrepeated sequence $D(I)$ and a string link $D_{I}(L)$ with $|D(I)|$ components, in a strictly similar way as for links in the introduction. By combining Corollary 6.3 and [26, Proposition 3.3] we have the following. 
Corollary 6.4 Two string links $L$ and $L^{\prime}$ cannot be distinguished by Milnor invariants if and only if they have same linking numbers and $f_{B_{D(I)}}\left(D_{I}\left(L \cdot \bar{L}^{\prime}\right)\right)=0$ for all sequences $I$.

\section{Example}

In this last section, we give a simple example illustrating the necessity of our hypothesis in Theorem 1.1.

Consider a link $L$ which is the split union of two positive Hopf links, with components labelled by 1,2 and 3, 4 respectively. Then, for the sequence $I=1324, \bar{\mu}_{L}(I)$ vanishes since $\Delta(I)=1$.

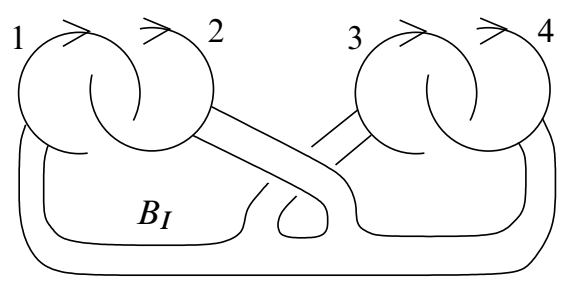

Figure 11

On the other hand, for the choice of $I$-fusion disk $B_{I}$ illustrated in Figure 11, we have

$$
P_{0}\left(L_{J}\right)= \begin{cases}2 t^{2}-t^{4} & \text { if } J=I, \\ 1 & \text { if } J \lesseqgtr I .\end{cases}
$$

Hence we notice that the alternate sum

$$
\sum_{J<I}(-1)^{|J|}\left(\log P_{0}\left(L_{J}\right)\right)^{(3)}=\sum_{J<I}(-1)^{|J|}\left(P_{0}\left(L_{J}\right)\right)^{(3)}=24
$$

is not divisible by $3 ! 2^{3}=48$.

This divisibility issue is the main obstruction for our formula to hold in general.

\section{References}

[1] D Bar-Natan, Vassiliev homotopy string link invariants, J. Knot Theory Ramifications 4 (1995) 13-32 MR1321289

[2] J C Cha, The effect of mutation on link concordance, 3-manifolds, and the Milnor invariants, J. Knot Theory Ramifications 15 (2006) 239-257 MR2207908 
[3] T D Cochran, Concordance invariance of coefficients of Conway's link polynomial, Invent. Math. 82 (1985) 527-541 MR811549

[4] C H Giffen, Link concordance implies link homotopy, Math. Scand. 45 (1979) 243-254 MR580602

[5] D L Goldsmith, Concordance implies homotopy for classical links in $M^{3}$, Comment. Math. Helv. 54 (1979) 347-355 MR543335

[6] M Gusarov, On n-equivalence of knots and invariants of finite degree, from: "Topology of manifolds and varieties", (O Viro, editor), Adv. Soviet Math. 18, Amer. Math. Soc. (1994) 173-192 MR1296895

[7] N Habegger, X-S Lin, The classification of links up to link-homotopy, J. Amer. Math. Soc. 3 (1990) 389-419 MR1026062

[8] N Habegger, G Masbaum, The Kontsevich integral and Milnor's invariants, Topology 39 (2000) 1253-1289 MR1783857

[9] K Habiro, Claspers and finite type invariants of links, Geom. Topol. 4 (2000) 1-83 MR1735632

[10] S Horiuchi, The Jacobi diagram for a $C_{n}$-move and the HOMFLY polynomial, J. Knot Theory Ramifications 16 (2007) 227-242 MR2306216

[11] T Kanenobu, $\mathbf{C}_{n}-$ moves and the HOMFLY polynomials of links, Bol. Soc. Mat. Mexicana 10 (2004) 263-277 MR2199353

[12] T Kanenobu, Y Miyazawa, HOMFLY polynomials as Vassiliev link invariants, from: "Knot theory (Warsaw, 1995)", (V F R Jones, J Kania-Bartoszynska, J H Przytycki, P Traczyk, V Turaev, editors), Banach Center Publ. 42, Polish Acad. Sci., Warsaw (1998) 165-185 MR1634455

[13] J Levine, A factorization of the Conway polynomial, Comment. Math. Helv. 74 (1999) 27-52 MR1677110

[14] W B R Lickorish, K C Millett, A polynomial invariant of oriented links, Topology 26 (1987) 107-141 MR880512

[15] X-S Lin, Power series expansions and invariants of links, from: "Geometric topology (Athens, GA, 1993)”, (W H Kazez, editor), AMS/IP Stud. Adv. Math. 2, Amer. Math. Soc. (1997) 184-202 MR1470727

[16] G Masbaum, A Vaintrob, Milnor numbers, spanning trees, and the AlexanderConway polynomial, Adv. Math. 180 (2003) 765-797 MR2020557

[17] J-B Meilhan, On Vassiliev invariants of order two for string links, J. Knot Theory Ramifications 14 (2005) 665-687 MR2162120

[18] J-B Meilhan, A Yasuhara, On $C_{n}$-moves for links, Pacific J. Math. 238 (2008) 119143 MR2443510

[19] J Milnor, Link groups, Ann. of Math. 59 (1954) 177-195 MR0071020 
[20] J Milnor, Isotopy of links. Algebraic geometry and topology, from: "A symposium in honor of S Lefschetz", (R H Fox, D C Spencer, A W Tucker, editors), Princeton Univ. Press (1957) 280-306 MR0092150

[21] K Murasugi, On Milnor's invariant for links, Trans. Amer. Math. Soc. 124 (1966) 94-110 MR0198453

[22] M Polyak, On Milnor's triple linking number, C. R. Acad. Sci. Paris Sér. I Math. 325 (1997) 77-82 MR1461401

[23] T Stanford, Braid commutators and Vassiliev invariants, Pacific J. Math. 174 (1996) 269-276 MR1398378

[24] L Traldi, Milnor's invariants and the completions of link modules, Trans. Amer. Math. Soc. 284 (1984) 401-424 MR742432

[25] L Traldi, Conway's potential function and its Taylor series, Kobe J. Math. 5 (1988) 233-263 MR990824

[26] A Yasuhara, Classification of string links up to self delta-moves and concordance, Algebr. Geom. Topol. 9 (2009) 265-275 MR2482076

[27] A Yasuhara, Self delta-equivalence for links whose Milnor's isotopy invariants vanish, Trans. Amer. Math. Soc. 361 (2009) 4721-4749 MR2506425

Institut Fourier, Université Grenoble 1

100 rue des Maths, BP 74, 38402 Saint-Martin d'Hères, France

Department of Mathematics, Tokyo Gakugei University

4-1-1 Nukuikita-Machi, Koganei-shi, Tokyo 184-8501, Japan

jean-baptiste.meilhan@ujf-grenoble.fr, yasuhara@u-gakugei.ac.jp

http://www-fourier.ujf-grenoble.fr/ meilhan/,

http://www.u-gakugei.ac.jp/ yasuhara/

Proposed: Peter Teichner

Seconded: Colin Rourke, Joan Birman
Received: 28 June 2011 Accepted: 2 February 2012 